\author{
Michele Barazzuol MD ${ }^{1}$, \\ Efthymia Lampropoulou MD ${ }^{1}$, \\ Andrea Meneghini $\mathrm{MD}^{2}$ and \\ Stefano Masiero $\mathrm{MD}^{1 \star}$ \\ ${ }^{I}$ School of Physical and Rehabilitation Medicine, \\ Department of Neurosciences, University of Padova, Italy \\ ${ }^{2}$ Advanced Technology in Rehabilitation Lab, Orthopedic \\ Rehabilitation Unit, Padova Hospital, Italy \\ Dates: Received: 01 November, 2014; Accepted: \\ 08 January, 2015; Published: 10 January, 2015 \\ *Corresponding author: Stefano Masiero MD, \\ School of Physical and Rehabilitation Medicine, \\ Department of Neurosciences, University of Padova, \\ Italy, E-mail: stef.masiero@unipd.it \\ www.peertechz.com
}

ISSN: 2455-5487

Keywords: Extracorporeal shockwave lithotripsy; Duchenne muscular dystrophy; Foot deformities

\section{Case Report \\ Extracorporeal Shock Waves in the Treatment of Equinovarus Foot in a Duchenne Patient: A Case Report}

\section{Introduction}

Duchenne muscular dystrophy (DMD) is the most frequent form of hereditary muscular dystrophy, affecting 1 in 3600-6000 live male births [1].

The disorder is characterized by a progressive muscular weakness especially of proximal muscle groups, with loss of independent ambulation. Children become wheelchair dependent when they are about 10 years, even earlier when patients are not treated with steroids $[2,3]$.

If untreated, the progression of the DMD can lead to grotesque deformities [4]. Equinus deformity of the ankle is one of the serious orthopedic problems associated with DMD [5]. The major cause of foot drop is the weakness of the muscles of ankle dorsiflexion, with a primary role supported by the deficiency of tibialis anterior, but also of the long extensors of the toes (extensor hallucis longus and extensor digitorum longus) [6]. A very important effect of this weakness is the shortening and contracture of the Achilles tendon. Most conditions that cause weakness of the dorsiflexors, may also affect the peroneus tertius, peroneus longus and tibialis posterior, respectively the muscles of eversion and inversion. The foot drop syndrome often incorporates weakness of these muscles, and associated contracture of their antagonist muscle tendons $s^{6}$. When ambulation is no longer possible, the rehabilitation program focuses on the prevention or reduction of complications and deformities, in order to alleviate pain and pressure, to allow the patient to wear shoes, and to correctly place the feet on wheelchair footrests [7].

Despite the absence of a specific treatment, the natural history of the DMD can be in part modified [1].

Since the surgical therapy showed controversial results on long term effectiveness, there has been a growing consideration on the conservative approach, including drugs and rehabilitation. Nevertheless, in most studies the rehabilitation programme includes stretching, eccentric and aerobic exercise, sub maximum strengthening activity when residual strength is higher, while few authors emphasize the role of physical energies, like whole-body vibration [8,9] and laser therapy [10].

Extracorporeal shock wave therapy (ESWT) is a relatively recent method used in the treatment of many musculoskeletal disorders, such as tendinopathies and non-union fractures.

Over the past few years, ESWT has received a widespread consensus in many other fields, including in the treatment of spastic hypertone in patients affected by stroke: even a single session of shock 
waves has proved to be effective in the reduction of muscle tone. Such effects are probably due both to a direct action on muscle and the production of several molecules and growth factors [11].

Therefore, we hypothesized that ESWT could have similar effects on dystrophic muscle.

\section{The Case}

A 20-year-old patient with bilateral equinovarus deformities (Figure 1) came to our ambulatory. At five years, he had the first difficulties during walking, getting a diagnosis of DMD at the age of seven.

A surgical correction was attempted by tendo-Achillis lenghtening on the left limb, at the age of nine; two years later, he required a permanent use of a wheelchair.

The patient had undertaken a rehabilitation program with an independently chosen physical therapist, which consisted of two sessions per week of passive mobilization of the four limbs, trunk and cervical spine.

At the time of our first evaluation, physical examination showed a complete loss of muscular strength in the upper and lower limbs: on ankle range of motion (ROM) assessment, active movements were not possible. Passive dorsiflexion was severely limited on both sides, with stronger restriction on the right foot (up to 8 degrees on the left and up to 5 degrees on the right side).

The right foot was oriented at $85^{\circ}$ of plantar flexion and $75^{\circ}$ of supination, while the left foot showed slightly lower alterations,

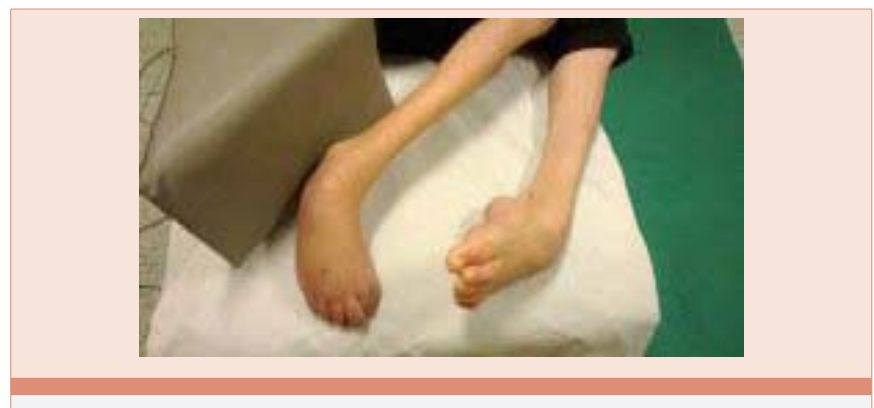

Figure 1: First assessment of foot deformities.

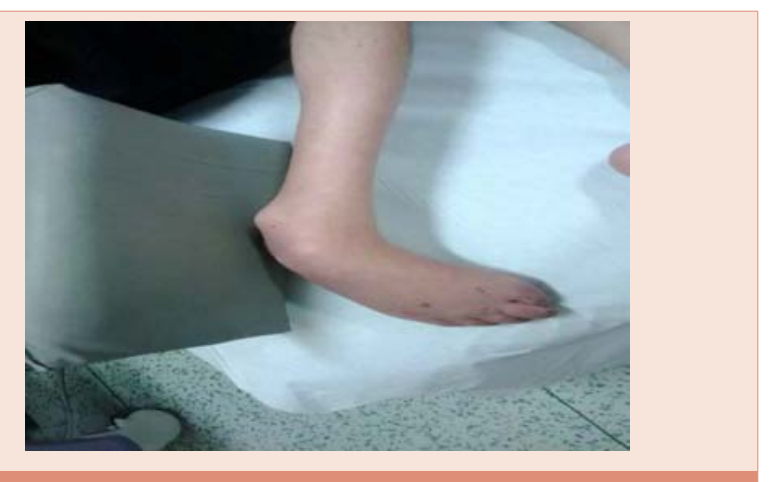

Figure 2: Right foot respectively before and after ESW treatment.

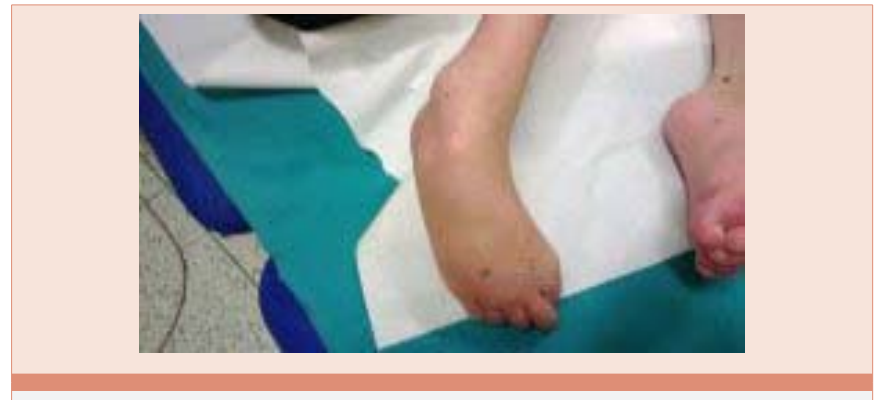

Figure 3: Right foot respectively before and after ESW treatment.

estimated respectively in $80^{\circ}$ and $65^{\circ}$. All measurements were performed with a goniometer by the same operator.

Furthermore, the skin of the feet appeared poorly vascularized and cold.

The deformities appeared very structured and with scarse margins of corrigibility, thus representing a great challenge.

Nevertheless, the patient was treated with gentle manipulation and casting as described by Ponseti [12] for two weeks, but no improvement in passive ROM was detected.

After this attempt, the treatment was modified by adding a session of low-energy ESWT: the new intervention was proposed for the subsequent four weeks.

The patient was treated immediately after removal of the former cast and the new one was applied within one hour, as suggested by Radler [13].

ESWT was performed by an electromagnetic shock wave system with echografic targeting (MODULITH' SLK, Storz Medical, Tägerwilen, Switzerland).

The schedule included 1200 shots applied to the soleus muscle and myotendinous junction, and 500 shocks applied to plantar flexors muscles, at energy flux density of $0.08 \mathrm{~mJ} / \mathrm{mm}^{2}$ and at frequency of 4 $\mathrm{Hz}$. Both limbs were equally treated.

\section{Results}

No pain was recorded during the treatment. The patient and his parents did not report any side effect after ESWT.

After five sessions of treatment (once a week), the physical examination revealed and a remarkable facilitation of passive mobilization of both feet.

The evaluation of limbs morphology showed a bilateral correction of the talo-metatarsal angle, more relevant on the right than on the left side, with an improvement respectively of $26^{\circ}$ and $19^{\circ}$ (Figures 2 and $3)$. However, the most considerable correction was assessed after only one session, when the talo-metatarsal angle showed an improvement of $13^{\circ}$ on the right and of $11^{\circ}$ on the left foot.

Both feet had a slightly less dramatic equinus deformity, with an improved orientation of $8^{\circ}$ on the right and $6^{\circ}$ on the left foot. The most relevant improvements were estimated in the passive dorsiflexion of both ankles: with a gentle manipulation, the operator 
was able to place both feet to the anatomical position, in such a way to allow the patient to wear his own shoes.

For the patient as well as for his family, this result represented an outstanding goal with favorable impact on the patient self-esteem and social relations.

\section{Discussion}

There is a widespread consensus that, in DMD patients, a multidisciplinary management is necessary, including pharmacological, rehabilitative, surgical and psychosocial interventions $[1,7]$.

Currently there is no curative therapy for this disease and the glucocorticoids are the only medication that can slow the loss of strength and muscle function in DMD [7].

In recent years, multiple treatment approaches have emerged for DMD. The need for novel therapeutic strategies for muscular dystrophies is particularly urgent.

Early surgery was very common in the past, but several studies showed to have a positive effect on Achilles tendon contractures only in the short-term, while long-term follow-up revealed recurrence of contractures in all patients who underwent intervention, demonstrating no functional benefit for the surgical approach alone [6].

Recently, the interest of many authors focused on emerging drugs for DMD: most promising are molecular-based therapies that can express the missing dystrophin protein or utrophin. Additional targets include the reduction of inflammation or the promotion of skeletal muscle blood flow and muscle contractility using nitric oxide (NO) donors [14].

The well-known regulation by $\mathrm{NO}$ of skeletal muscle force excitation-contraction coupling and the newly characterized role of $\mathrm{NO}$ in myogenesis and muscle repair, suggest a possible use of NO as a tool for therapeutic strategies in DMD [15].

In skeletal muscle a lack of dystrophin determines the suspension of neuronal NOS (nitric oxide synthase) action to the sarcolemma, thus affecting NO (nitric oxide) production [16].

In addition to this, there is growing evidence that indicate how the dystrophic muscle is under a condition of vascular abnormalities and ischemia.

Although some studies show that blood VEGF levels are significantly lower in DMD, other authors emphasize the inconsistency of these data, nonetheless suggesting a fluctuation of VEGF secretion according to age or disease progression. DMD muscle may benefit from angiogenesis in multidimensional aspects: increasing capillary density, which directly resolves ischemia; regenerative and antiapoptotic effects of VEGF; proliferation of satellite cells [17].

One of the confirmed effects after ESWT is a rapid increase in blood flow around the treated area. This observation led to the assumption that ESWT may directly involve the production of NO, one of the strongest endogenous vasodilatators known up to date [18].
Other studies have focused on the role of shock waves in inducing neovascularization and increasing of angiogenesis and osteogenesis related growth factors, such as eNOS (endothelial nitric oxide synthase), VEGF, PCNA (proliferating cell nuclear antigen) and BMP-2 (bone morphogenic protein 2) [19]. ESW may modulate endogenous NO production either under normal or inflammatory conditions.

An increase in VEGF is an indication of increased vascular permeability and microvascular activity, including angiogenic growth of new blood vessels. ESWT- promoted bone healing was associated with significant elevations of VEGF and angiogenesis and osteogenesis growth factors, whereas, inhibition of VEGF attenuated the effects of ESWT and decreased angiogenesis and osteogenesis factors [19].

The use of physical therapy in the treatment of DMD is a current issue.

In the past few years, some studies focused on whole-body vibration (WBV), showing that the WBV exercise did not further damage the skeletal muscle and caused a non-significant increase in bone formation, the meaning of which remains unclear [8].

WBV training seems to have a role in a transient production of creatine kinase, but the relevance of the temporary increase in creatine kinase in DMD during the first days of training is not yet understood [9].

Low-level laser therapy (LLLT) has a protective function on damaged or dystrophic skeletal muscle against degeneration, modulating inflammatory citokines and up-regulating mitochondrial activity, with the result of a decreased oxidative stress [10].

Many factors have been considered to explain the effects of ESWT on spastic muscles. NO synthesis has been suggested to be one of the most physiologically important mechanisms that could explain the effectiveness of shock waves in the anti-inflammatory treatment of tendinopathies, but a direct effect of shock waves on hypertonic muscle should be considered, as well as a direct effect on the muscle fibers adjacent to the tendon cannot be excluded [11].

Finally, ESWT and WBV stimulation show many differences in efficacy duration and clinical responses, suggesting a different mechanism of action [11].

To our knowledge, only one preliminary study includes ESWT within a rehabilitation program in patients with DMD [20]: at the end of the treatment, stability of motor skills, horizontal and vertical enhancement of the capacity have been reported.

\section{Conclusions}

This case confirms that low-energy ESWT is safe and unpainful, and suggests a potential corrective action on foot deformities in patients affected by DMD. In less advanced cases, the use of shock waves might be considered part of an integrated approach to the rehabilitation program.

The treatment was intended to reduce the contraction of the antagonist muscles and improve the blood supply of the lower limb, without any target on the recovery of muscular force. 
However, even the simple act of wearing the shoes had a significant impact on the degree of patient satisfaction and its quality of life.

\section{Limitations}

Our case is purely anecdotal: the patient was in a very advanced condition. Data on the duration of ESWT effectiveness are not available: due to a worsening of respiratory conditions, the patient could not perform the scheduled follow-up after 3 and 6 months, so that the evalutation of long term effectiveness is lacking.

Further studies including a larger sample size, possibly at earlier stage of DMD, are requested to confirm a potential role of shock waves in the prevention and treatment of foot deformities in Duchenne patients.

\section{References}

1. Bushby K, Finkel R, Birnkrant DJ, Case LE, Clemens PR, et al. (2010) Diagnosis and management of Duchenne muscular dystrophy, part 1: diagnosis, and pharmacological and psychosocial management. Lancet Neurol 9: 77-93

2. Bushby KM, Hill A, Steele JG (1999) Failure of early diagnosis in symptomatic Duchenne muscular dystrophy. Lancet 353: 555-578.

3. Poysky J (2007) Behavior in DMD Study Group. Behavior patterns in Duchenne muscular dystrophy: report on the Parent Project Muscular Dystrophy behavior workshop 8-9 of December 2006, Philadelphia, USA. Neuromuscul Disord 17: 986-994.

4. Dubowitz V (2010) Deformities in Duchenne dystrophy. Neuromuscul Disord 20: 282.

5. Williams EA, Read L, Ellis A, Morris P, Galasko CS (1984) The management of equinus deformity in Duchenne muscular dystrophy. J Bone Joint Surg $\mathrm{Br}$ 66: $546-550$

6. Sackley C, Disler PB, Turner-Stokes L, Wade DT, Brittle N, et al. (2009) Rehabilitation interventions for foot drop in neuromuscular disease. Cochrane Database Syst Rev CD003908.

7. Bushby K, Finkel R, Birnkrant DJ, Case LE, Clemens PR, et al. (2010) Diagnosis and management of Duchenne muscular dystrophy. Part 2: Implementation of multidisciplinary care. Lancet Neurol 9: 177-189.

8. Vry J, Schubert IJ, Semler O, Haug V, Schönau E, et al. (2014) Whole-body vibration training in children with Duchenne muscular dystrophy and spinal muscular atrophy. Eur J Paediatr Neurol 18: 140-149.

9. Söderpalm AC, Kroksmark AK, Magnusson P, Karlsson J, Tulinius M, et al. (2013) Whole body vibration therapy in patients with Duchenne muscular dystrophy--a prospective observational study. J Musculoskelet Neuronal Interact. 13: 13-18.

10. Ferraresi C, Hamblin MR, Parizotto NA (2012) Low-level laser (light) therapy (LLLT) on muscle tissue: performance, fatigue and repair benefited by the power of light. Photonics Lasers Med 1: 267-286.

11. Manganotti P, Amelio E (2005) Long-term effect of shock wave therapy on upper limb hypertonia in patients affected by stroke. Stroke 36: 1967-1971.

12. Ponseti IV, Campos J (1972) Observations on pathogenesis and treatment of congenital club foot. Clin Orthop Relat Res 84: 50-60.

13. Radler C (2013) The Ponseti method for the treatment of congenital clubfoot: review of the current literature and treatment recommendations. Int Orthop 37: 1747-1753.

14. Malik V, Rodino-Klapac LR, Mendell JR (2012) Emerging drugs for Duchenne muscular dystrophy. Expert Opin Emerg Drugs 17: 261-277.

15. De Palma C, Clementi E (2012) Nitric Oxide in Myogenesis and Therapeutic Muscle Repair. Mol Neurobiol 46: 682-692.

16. Ramachandran J, Schneider JS, Crassous PA, Zheng R, Gonzalez JP, et al. (2013) Nitric oxide signalling pathway in Duchenne muscular dystrophy mice: up-regulation of L-arginine transporters. Biochem J 449: 133-142.

17. Shimizu-Motohashi Y, Asakura A (2014) Angiogenesis as a novel therapeutic strategy for Duchenne muscular dystrophy through decreased ischemia and increased satellite cells. Front in Physiol 5: 50.

18. Mariotto S, de Prati AC, Cavalieri E, Amelio E, Marlinghaus E, et al. (2009) Extracorporeal shock wave therapy in inflammatory diseases: molecular mechanism that triggers anti-inflammatory action. Curr Med Chem 16: 23662372.

19. Wang CJ, Huang KE, Sun YC, Yang YJ, Ko JY, et al. (2011) VEGF Modulates Angiogenesis and Osteogenesis in Shockwave-Promoted Fracture Healing in Rabbits. J Surg Res 171: 114-119.

20. Marinò C, Corrado B, Servodio lammarrone C (2002) ESWT: a Real Help in the Rehabilitation Protocol of Patients Affected by Duchenne Muscular Dystrophy - a Preliminary Report. In: The 5th International Congress of the ISMST, 26-29 June, Winterthur.

Copyright: (c) 2015 Barazzuol M, et al. This is an open-access article distributed under the terms of the Creative Commons Attribution License, which permits unrestricted use, distribution, and reproduction in any medium, provided the original author and source are credited. 